\title{
Imaging the stimuli response of nanostructured materials inside a transmission electron microscope: from today's sub-second recording to ultrafast phenomena
}

\author{
P.M.F.J. Costa**** \\ *CICECO, Departamento de Engenharia de Materiais e Cerâmica, Universidade de Aveiro, 3810-193 Aveiro, \\ Portugal \\ **Leibniz Institute for Solid State and Materials Research (IFW), 01069 Dresden, Germany
}

Over the last decade, with the advent of aberration correctors and energy-filters, transmission electron microscopes (TEMs) have seen phenomenal developments both in regards to spatial and spectral resolutions. Today, the TEM is a characterisation tool pervasive to nanoscaled sciences and technologies. Imaging and identifying single atoms, including low $\mathrm{Z}$ elements such as $\mathrm{B}$ or $\mathrm{C}$, is within reach of a skilful operator with access to this new generation of microscopes.

Parallel to these advances, sample holders have also been evolving. Instead of just fixing and orienting the sample for observation, these ancillary components of TEMs have gradually been morphing into full-fledged instruments. Increasingly sophisticated devices enable a multitude of experiments performed inside the column of the TEM which includes the manipulation and exposure of discrete nanostructures to a variety of stimuli (heat, pressure, electrical current...) [1]. An example is the real-time observation of solid-vapour phase transitions in filled carbon nanotubes acting as interconnects and experiencing the dynamical effects of Joule heating [2]. Together with resolution improvements, these added capabilities represent a paradigmatic shift in how we perceive and work with TEMs. No longer is the electron microscopist limited to simply analyse specimens in "post-mortem" state but (s)he can now take hold of a full set of physical and chemical experiments that encompass a much broader range of scientific interests.

Despite all progress, there is a crucial part of the TEM capabilities that is still awaiting a similar leap in resolution: the time-domain. In result of the past dominance of "post-mortem" analysis, a temporal resolution enhancement has been overlooked by most instrument manufacturers and researchers. However, with the growing interest on in situ methodologies, the ability to perform time-resolved experiments has suddenly become a fundamental necessity. Classically, registering tools such as photographic films, charge-coupled devices or video-rated cameras have been adequate to acquire data on events that take place on timescales ranging from seconds to hours. Such temporal resolution is not sufficient for an immensity of life- and materials science-related processes. To illustrate this, Figure 1 shows the before and after states of a ZnS-filled carbon nanotube that was subjected to a pulse of electrical current [3]. Although it is possible to observe clearly the end result, one can only speculate on how the confined semiconductor was released, transported and delivered to the receiving substrate. Considering that the pulse lasted for $250 \mathrm{~ms}$, a temporal resolution of at least $50 \mathrm{~ms}$ (or 20 frames per second) for the registering device would be necessary to build a reasonable impression of the mechanism behind this delivery-procedure.

\section{References}

1. Golberg D. et al., Adv. Mater., 24:177, 2012.

2. Costa P.M.F.J. et al., Nat. Commun., 2:421, 2011.

3. Costa P.M.F.J. et al., Carbon, 49:3747, 2011. 


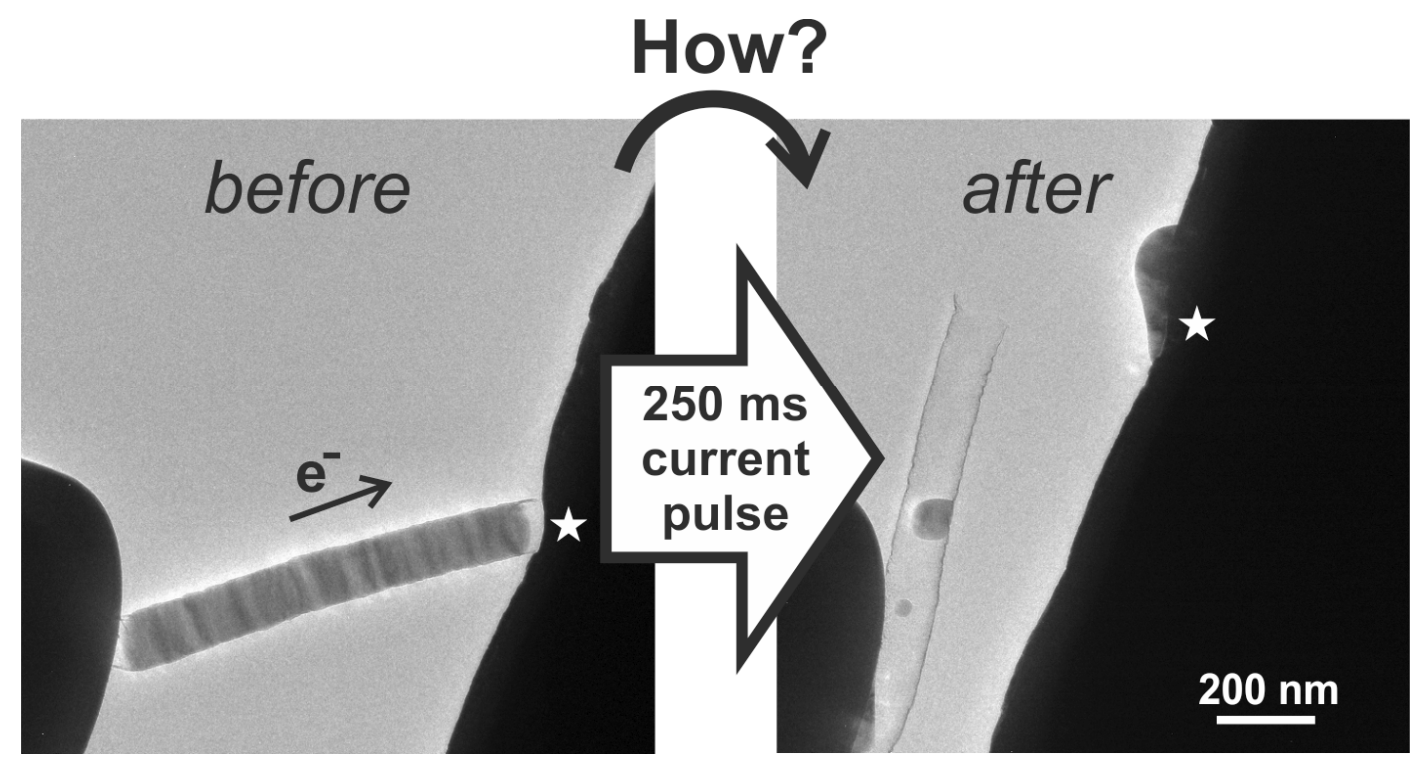

Figure 1. Following careful manipulation within the TEM, a short carbon nanotube entirely filled with Ga-doped $\mathrm{ZnS}$ is placed between two $\mathrm{Au}$ electrodes and a high current density pulse is injected into it. Most of the confined semiconductor mass is instantaneously expelled with part of it delivered on the receiving substrate (cf. star in both panels).

PMFJC acknowledges the support from the Fundação para a Ciência e Tecnologia (Ciencia2007 Fellowship and project grant PTDC/EME-PME/112073/2009) and Alexander von Humboldt Foundation (Advanced Researcher Fellowship). 